\title{
Aportaciones de las universidades on-line al sistema universitario
}

\section{El gran mérito de la formación a distancia ha consistido en el aprovechamiento de cualquier avance tecnológico que permitiera un replanteamiento metodológico de la formación superior, con el fin de mejorar la difusión de contenidos y el acercamiento del profesorl tutor a sus estudiantes}

Las universidades virtuales, al igual que el resto de las instituciones de educación superior presenciales, se apoyan en cuatro grandes pilares:

- La producción del conocimiento, a través, principalmente, de la investigación.

- Su transmisión mediante la educación y la formación.

- Su difusión a través de las técnicas de la información y la comunicación.

- El uso de dichas técnicas en la innovación tecnológica.

En tal sentido, su aportación al sistema universitario español es el general de toda institución de formación superior, no obstante lo cual presentan rasgos destacables que conviene enfatizar, porque suponen una especial contribución a ese acervo común. Uno de esos rasgos distintivos es el uso intensivo de las tecnologías de la información y la comunicación (TIC), cuyas funcionalidades se conocen como

\footnotetext{
J. A. Sánchez Pedroche, rector de la Universidad a Distancia de Madrid (UDIMA).

2 El texto corresponde a la conferencia pronunciada en el Centro Riojano de Madrid, el día 3 de marzo de 2016, en el «ll Encuentro de Universidades On-line. Propuestas para el Futuro de la Educación Universitaria On-line», organizado por la Universidad Nacional de Educación a Distancia (UNED), la Universitat Oberta de Catalunya (UOC), la Universidad Internacional de Valencia (VIU), la Universidad Internacional de La Rioja (UNIR), la Universidad Isabel I y la Universidad a Distancia de Madrid (UDIMA).
} 


\section{El profesor, en un entorno virtual, se convierte en el dinamizador y facilitador del proceso de aprendizaje de sus estudiantes, lo que le obliga a entenderlos, a acompañarlos en ese nuevo contexto formativo, mucho menos normativo $y$, por ello, más abierto $y$ libre, pero también más inseguro}

triple A [anywhere (cualquier lugar), anytime (cualquier hora) y anyone (cualquier persona)], aunque ciertamente no constituyan un objetivo en sí mismas, siendo simplemente un mecanismo para alcanzar determinados objetivos de aprendizaje específicos. Ahora bien, no puede perderse de vista que los nuevos entornos de aprendizaje no dependen tanto de ellas, cuanto del cambio de paradigma metodológico, capaz a su través de lograr unas metas formativas distintas a las tradicionales.

El gran mérito de la formación a distancia ha consistido en el aprovechamiento de cualquier avance tecnológico que permitiera un replanteamiento metodológico de la formación superior, con el fin de mejorar la difusión de contenidos y el acercamiento del profesor/tutor a sus estudiantes. En otras palabras: la rápida capacidad de reacción y adaptación de las universidades on-line a las nuevas y cambiantes necesidades formativas, toda vez que el verdadero modelo de conocimiento en las universidades virtuales va más allá de la transmisión pasiva de conocimientos, porque no se piensa en el estudiante como una «caja vacía que deba llenarse», sino más bien «como una hoguera que necesariamente hay que encender». No creo equivocarme si les señalo el importante papel que la formación a distancia ha prestado y viene prestando al sistema universitario español, si bien estoy persuadido de que esa relevancia será mucho más evidente en el futuro por varias razones a las que enseguida me referiré. La UNED y la UOC fueron las pioneras en este campo, con unas aportaciones docentes y científicas muy relevantes, así como con un número de estudiantes especialmente significativo.
La principal razón de ser de las universidades virtuales no era otra que acercar la formación superior a personas que no tenían la oportunidad de acceder a una universidad presencial por razón de su lugar de residencia u ocupación. Así, tradicionalmente la formación a distancia ha venido cumpliendo una evidente función social de mejora del nivel de formación general del país, contribuyendo de esta manera a atemperar las barreras existentes para acceder a los recursos y oportunidades de la enseñanza superior, las cuales privan a los estudiantes de posibilidades de desarrollo a su alcance (recursos de aprendizaje, materiales de referencia, ejercicios de autoevaluación, herramientas de comunicación interactivas y un largo etcétera). Todo esto es particularmente cierto para los segmentos de población estudiantil que viven en áreas geográficamente alejadas, así como para los menos favorecidos en términos físicos, organizativos, sociales o económicos. Ahora bien, en la actualidad dicha función social cobra, si cabe, mayor relevancia cuando las necesidades de formación son extraordinariamente cambiantes y los profesionales precisan de una oferta formativa permanente.

Tradicionalmente el profesor universitario se limitaba a ser un experto en su área de conocimiento, mientras que sus competencias pedagógicas las adquiría con la experiencia 0 , en el mejor de los casos, por imitación de algunos excelentes profesionales de la formación superior. Resulta relativamente reciente la preocupación por esa imprescindible preparación pedagógica y la evaluación de la calidad docente en la formación universitaria. Este esfuerzo se ha ido imponiendo, aunque no sin resistencia, a fuerza de evidenciar las necesidades de mejora de los distintos sistemas educativos. Sin embargo, en el caso de la formación universitaria on-line, el hecho de enfrentarse constitutivamente a un entorno y a unas metodologías distintas y especiales ha facilitado la predisposición de las propias instituciones a situar la formación de sus docentes como prioridad estratégica, ofreciéndoles las claves de análisis y reflexión sobre la mejor forma de facilitar el aprendizaje de sus estudiantes. De la misma manera, la formación on-line requiere, por definición, una visión activa del alumno en la evolución de su aprendizaje, motivo por el cual en ese contexto no han sido necesarios procesos especiales de transformación que huyeran de la clase magistral y de un enfoque exclusivamente conceptual. 
La necesidad de enfrentarse a un nuevo entorno formativo, es decir, a un paradigma distinto (ese paso de la enseñanza al aprendizaje), obliga al docente virtual a exigirse de una forma natural aquellos aspectos considerados ejes fundamentales de la calidad de la enseñanza, recogidos ya, por ejemplo, en los principios del espacio europeo de educación superior (EEES). Dicho de otra manera, la propia idiosincrasia de la formación on-line hace que algunos retos de transformación del modelo educativo de enseñanza superior -recogidos en las directivas del EEESse conciten de forma absolutamente natural en las universidades virtuales.

Como ha señalado el profesor de la Universidad de Stanford, G. Kembel, nuestro sistema educativo se ha basado en la transferencia a los estudiantes de lo que sabemos, y la forma de hacerlo no ha sido otra que a través de las clases magistrales. Con los exámenes se evalúa si los alumnos contestan 0 no lo correcto. Pero ese método tradicional ya no sirve, o al menos no en la misma medida en que lo hacía antes. Ignoramos los trabajos que existirán dentro de unos pocos años y desconocemos igualmente lo que los estudiantes tendrán que resolver.

\section{(...) la propia idiosincrasia de la formación on-line hace que algunos retos de transformación del modelo educativo de enseñanza superior -recogidos en las directivas del EEES- se conciten de forma absolutamente natural en las universidades virtuales}

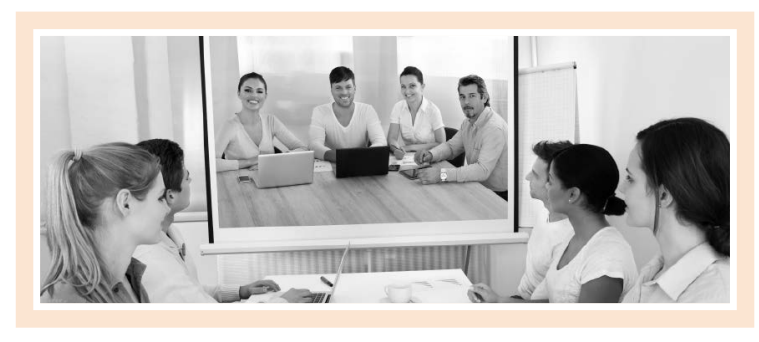

En el mundo actual, todo cambia deprisa, y si alguna certeza existe no es otra que la necesidad de entrenar a las mentes para saber reaccionar frente a la incertidumbre. No se aprende ya escuchando a un profesor, sino indagando, enfrentándose a disyuntivas y acometiendo proyectos reales; detectar problemas, diseñar soluciones y probarlas en la práctica. La clave no está tanto en resolver esos problemas, cuanto en advertir de su existencia. Los profesores, que vienen de las diferentes facultades de Stanford y de empresas innovadoras, afirma Kembel, actúan como guías y aprenden con los estudiantes. Esa es la gran diferencia. $\mathrm{Y}$ claro, las funciones del profesorado en ese contexto son ya otras. No se trata de insistir en un conocimiento que se puede adquirir mucho mejor y más abundantemente en la red que en la clase magistral. Se trata, por el contrario, de diseñar acciones formativas vinculadas a entornos o ambientes de aprendizaje capaces de incorporar elementos de valor añadido: multiplicidad de recursos formativos y de investigación, herramientas colaborativas, mecanismos de comunicación individual y múltiple, sistemas de autoevaluación y retroalimentación, trabajo en equipo y un largo etcétera. Justo lo que ya vienen haciendo las universidades virtuales por imperiosa necesidad.

Ese enorme potencial de la formación on-line fue advertido tempranamente por Gerard Gaspar, expresidente de la Universidad de Stanford -en cuyo mandato eclosionaron herramientas e ideas empresariales con repercusión planetaria como Google o Sun Microsystem Corporation-, quien abogó por la virtualización de los campus de las universidades presenciales como revulsivo imprescindible a ese cambio de paradigma de la enseñanza al aprendizaje. Instituciones tan prestigiosas como el Massachusetts Institute of Technology (MIT) pronto abordaron también proyectos virtuales intuitivos y alejados de la aparatosidad como los Open Course Ware. Inútil es insistir en el hecho de la cooperación universitaria a través de las TIC, a pesar de algunas experiencias fallidas como $\mathrm{FATHOM}^{3}$, pues las mismas son hoy en día una pujante realidad.

\footnotetext{
${ }^{3}$ Impulsada por Columbia University, Chicago University, Michigan University, London School of Economics, British Museum, New York Public Library y Cambridge University Press, entre otras.
} 


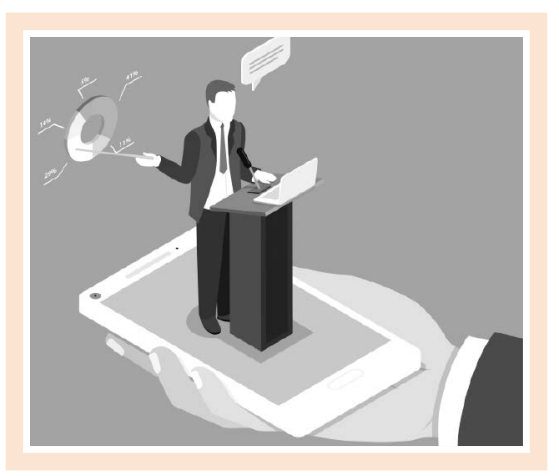

El profesor, en un entorno virtual, ve reforzado su papel como el verdadero gestor de una comunidad de aprendizaje, y en ese contexto relacional los roles se intercambian entre todos sus agentes, por cuanto el conocimiento ya no reside exclusivamente en quien aparece como profesor, sino que cada uno de dichos agentes actúan en conjunto, generando a su vez un foco de creación de nuevo conocimiento. El profesor, además de un dinamizador de su comunidad educativa, es un ingeniero pedagógico, pues ha de reorientar su objetivo didáctico en forma de metainformación añadida al recurso destinado a la construcción del objeto de aprendizaje. Interpretar elementos de información compatibles y entregarlos a los estudiantes a través de las TIC incide directamente en la necesidad de las respectivas organizaciones de compartir información educativa aprovechando los recursos de manera óptima, así como facilitando la cooperación interuniversitaria.

En las aulas virtuales se exige disponer de habilidades organizativas más intensas, que abjuran un tanto de ese formato bidireccional convencional en beneficio de otros enfoques y otras herramientas de trabajo, síncronas 0 asíncronas. Como consecuencia de la planificación del aprendizaje, las actividades orientadas a este fin cobran mayor protagonismo y se convierten en la piedra angular del proceso de enseñanza y aprendizaje. El peso se traslada del contenido a la actividad, del conocimiento mismo a la construcción de dicho conocimiento y al desarrollo y adquisición de competencias.
Capacidad y habilidad adaptativa de la metodología aplicada a la enseñanza superior es una característica intrínseca a la formación virtual, así como la predisposición continua al cambio, inherente a la nueva sociedad de la información. El profesor, en un entorno virtual, se convierte en el dinamizador y facilitador del proceso de aprendizaje de sus estudiantes, lo que le obliga a entenderlos, a acompañarlos en ese nuevo contexto formativo, mucho menos normativo y, por ello, más abierto y libre, pero también más inseguro. Lo anterior exige además que el profesor se oriente a la adecuada comprobación o constatación de que el estudiante incorpora ese aprendizaje de forma adecuada. El mundo virtual y las TIC exigen un enfoque holístico en el desarrollo de la formación del profesorado que no se limita ni mucho menos al carácter tecnológico, sino que exige una visión sistemática de mayor alcance. Y es que con la formación virtual se activa una metodología conducente a la consecución de una serie de objetivos tan relevantes como los siguientes:

- La orientación hacia el descubrimiento de analogías entre fenómenos diferentes.

- La proposición de actividades que, fomentando la iniciativa personal, exijan planteamientos totales e individuales a través de la cooperación y el uso de las TIC y donde además se suscite la discusión con el fin de que el estudiante aporte razones que hagan prevalecer una tesis sobre otra.

- El establecimiento de relaciones entre el tema objeto de estudio y la realidad.

- La proposición de trabajos libres, siempre relacionados con el objeto de estudio, pero sobre los que el estudiante pudiera encontrarse más motivado 0 interesado.

Para que un estudiante se vea movido a
aprender algo nuevo, ese conocimiento
debe revestir dos condiciones
imprescindibles: interés y sentido. Es
lo que se conoce como uaprendizaje
significativo», que no solo favorece ese
proceso con mucho menor esfuerzo,
sino que además ayuda al estudiante a
reconstruir de manera activa sus propios
conocimientos

Para que un estudiante se vea movido a aprender algo nuevo, ese conocimiento debe revestir dos condiciones imprescindibles: interés y sentido. Es lo que se conoce como uaprendizaje significativo", que no solo favorece ese proceso con mucho menor esfuerzo, sino que además ayuda al estudiante a reconstruir de manera activa sus propios conocimientos 
En este nuevo contexto al que acabo de referirme el profesor ha de asumir que ya no puede limitarse a ser un mero dispensador de conocimientos, sino que debe convertirse en una herramienta capaz de acompañar y tutelar el proceso de aprendizaje de sus estudiantes. Sin motivación no hay aprendizaje, y sin ambos se genera la deserción y el abandono. Para que un estudiante se vea movido a aprender algo nuevo, ese conocimiento debe revestir dos condiciones imprescindibles: interés y sentido. Es lo que se conoce como «aprendizaje significativo», que no solo favorece ese proceso con mucho menor esfuerzo, sino que además ayuda al estudiante a reconstruir de manera activa sus propios conocimientos al encontrarse con otros nuevos. En este aprendizaje significativo, propio de la formación no presencial, cada estudiante se siente protagonista y responsable de su propio proceso educativo porque sabe lo que quiere lograr y conoce en cada momento lo que debe hacer y la finalidad con que lo hace ${ }^{4}$.

Todo lo anterior debe relacionarse también con una nueva metodología de aprendizaje que a buen seguro cobrará mucha entidad en el futuro más próximo. Me refiero a los cursos abiertos masivos [massive open on-line course (MOOC)]. Con ellos el mapa de las titulaciones cambiará con toda seguridad, permitiéndose -no tendría sentido lo contrario- que al menos un buen número de créditos puedan cursarse 0 convalidarse con cursos de las más prestigiosas universidades del mundo a elección plena y libre de cualquier estudiante. En tal caso, y una vez dilucidada la metodología para evaluar correctamente el aprovechamiento de dichos cursos, el ministerio o las universidades españolas habrán de convalidar la realización de esos MOOC y el profesor deberá reorientar necesariamente su papel hacia un servicio de tutorización y acompañamiento a los estudiantes que opten por dichos cursos en abierto; con un efecto añadido: dicha tutorización le obligará a un esfuerzo de excelencia que redundará a buen seguro en la mejora de todo el proceso interno universitario.

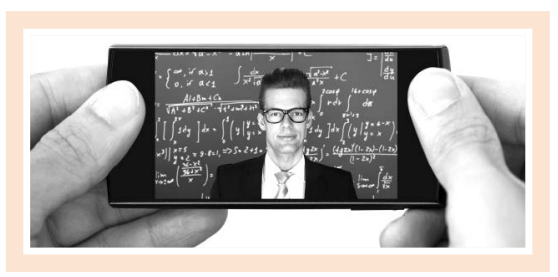

Por todo ello, la contribución de las universidades virtuales al sistema de formación superior común español es más que evidente en el campo de la docencia, toda vez que las competencias de su profesorado constituyen un ejemplo elocuente en los necesarios cambios que deben acometerse a nivel general, sobre todo si no se olvida que estar a la altura de las exigencias del Proceso de Bolonia constituye uno de los grandes retos de la universidad española actual; un desafío, por cierto, todavía inconcluso.

Pero competir en el ámbito global también constituye un reto ineludible. La internacionalización del sistema universitario español reclama una atención impostergable y, entre las iniciativas tomadas en esta dirección, cabe destacar la Estrategia Universitaria 2015, cuyos tres principales objetivos (acordados por el ministerio y las universidades) fueron los siguientes:

- Atraer a buenos alumnos del ámbito internacional.

- Ofrecer incentivos para que los profesores e investigadores mejoren su carrera académica.

- Situarse en vanguardia en la investigación de las principales ramas del saber.

\footnotetext{
4 Ahora bien, esto no encierra una visión moderna o novedosa del proceso educativo. Educar proviene del verbo educere, que en latín clásico significó primariamente «sacar» 0 «desenvolver». Eso que se saca o desenvuelve son las ideas y los valores que están dormidos, en el fondo de uno mismo, y que el maestro tiene que reactivar o reavivar, esto es, sacar a la luz. Nada mejor, para hacerse una idea clara de tal proceso, que acudir al Menón platónico. La pregunta que da origen al diálogo no es otra que la que el propio Menón le hace a Sócrates: si la areté puede enseñarse. Para responder a esta pregunta, Sócrates recurre a un esclavo, absolutamente iletrado, del propio Menón. Tras someterle a un hábil interrogatorio, el esclavo acaba razonando como el mejor geómetra, lo cual demuestra que él ya sabía esas grandes verdades de la geometría, por más que no fuera consciente de ello. La función del maestro es, pues, sacar a la luz eso que todos llevamos dentro, ya que las ideas son esencias puras que todos hemos visto en una vida anterior y de las que guardamos memoria. Así, el aprendizaje es un proceso de reminiscencia y el maestro un estricto educador, es decir, alguien que consigue hacer explícito lo implícito, que extrae del interior de cada uno esas ideas puras o esencias objetivas que existen independientemente de nosotros y de las que guardamos oscura memoria. La función del profesor no es otra que trasparecer lo escondido o sacar a la luz lo recóndito. De ahí que la formación no tenga otro objeto que educar, es decir, sacar, desplegar. De lo que se trata es de que el discípulo descubra esos valores que se hallan presentes desde siempre en el fondo de su alma. Hasta tal punto esta idea está enraizada en los clásicos que Platón afirmará en su Menón que los valores no se enseñan, sino que se recuerdan en su bien conocida doctrina de la anamnesis: «Afirmo que no hay enseñanza (didajèn) sino recuerdo (anámnesin)». Cfr. Menón, 1970, Madrid, Instituto de Estudios Políticos, pág. 82.
} 


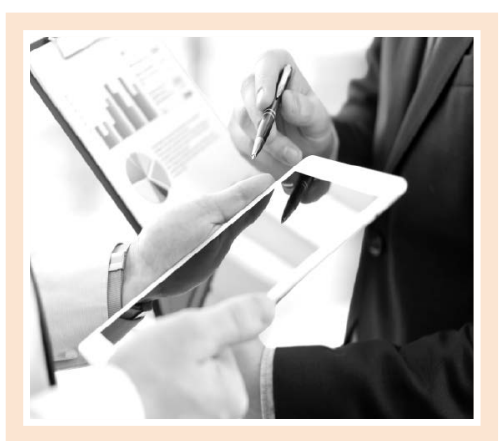

Aunque la iniciativa se dirige, sin duda, en la buena dirección, la universidad española todavía está lejos de conseguir tales objetivos. No voy a referirme a esa perniciosa e inveterada endogamia que cierra las puertas no solo al talento internacional, sino incluso al interno. Tampoco lo haré a la baja calidad en la enseñanza, modulada por la excesiva carga administrativa de los profesores, un sistema retributivo que estimula muy poco la actividad docente e investigadora, aulas muy numerosas, escaso contacto entre profesor y alumno y un sistema excesivamente basado en exámenes finales (por no hablar de la falta de especialización y la casi nula relación entre el mundo universitario y el de la empresa). Sí quisiera centrarme, sin embargo, en la escasa proyección internacional del sistema universitario español.

Los principales signos de ese problema son tres. En primer lugar, son muy escasos los alumnos extranjeros en nuestras aulas universitarias; en segundo término, hay muy pocos incentivos para lograr que académicos españoles en el exterior regresen a nuestro país para aportar su docencia e investigación; y, por fin, hay pocos profesores extranjeros dispuestos a insertarse en los claustros universitarios nacionales debido a la endogamia y a las formidables dificultades burocráticas para acceder a la carrera académica en España a las que antes me he referido.
El «Times Higher Education Supplement» sobre las 200 mejores universidades del mundo, uno de los informes de mayor reputación en el ámbito universitario, elabora un ranking anual sobre universidades basado en los siguientes criterios:

- Calidad de la enseñanza.

- Investigación.

- Repercusión de dicha investigación en la sociedad.

- Rentabilidad industrial.

- Alumnado internacional.

Aun valorando la subjetividad y el sesgo inherente a estos informes y a sus resultados -dichos rankings, que no necesariamente prestan atención a todos los factores que contribuyen a la excelencia académica-, no queda otro remedio que asumirlos y tenerlos en cuenta, debido a su relevancia internacional. España tiene un porcentaje muy bajo de estudiantes extranjeros. De los 3,4 millones de alumnos que estudian fuera de sus países de origen en el mundo, la universidad española solamente atrae al $1,4 \%$. En primero y segundo ciclo, el porcentaje es de apenas un $3,6 \%$ de universitarios extranjeros, frente al $8010 \%$ de la media europea. Por lo tanto, para competir en el ámbito global, es necesario saber incentivar a los estudiantes internacionales y atraerlos al sistema universitario patrio. En algunas universidades se han asignado presupuestos específicos para captar profesores de excelencia internacionales, pero estas iniciativas han fracasado no solo porque no se ha podido asignar una retribución equiparable a las propias del mercado internacional, sino también porque no se garantizaba una estabilidad laboral razonable, amén de la necesidad de trasladarse físicamente a España.

De los 3,4 millones de alumnos que estudian fuera de sus países de origen en el mundo, la universidad española solamente atrae al $1,4 \%$. En primero y segundo ciclo, el porcentaje es de apenas un $3,6 \%$ de universitarios extranjeros, frente al 80 $10 \%$ de la media europea. Por lo tanto, para competir en el ámbito global, es necesario saber incentivar a los estudiantes internacionales $y$ atraerlos al sistema universitario patrio 
Alemania y los Países Bajos han logrado internacionalizar su sistema universitario mediante una hábil combinación de la enseñanza en su lengua autóctona y el inglés, una estrecha colaboración con el mundo empresarial y una alta dosis de especialización. La estrategia UE-15 ha propuesto un conjunto de medidas que se encaminan en la dirección adecuada. Sobre todo, aumentar la internacionalización de la oferta universitaria con acciones tendentes a ofertar titulaciones interuniversitarias e internacionales, contratar profesores universitarios en el concierto internacional, incentivar cursos en lenguas foráneas (inglés y francés, fundamentalmente) 0 crear doctorados de referencia internacional.

El grado de compromiso con estos objetivos por parte del Ministerio de Educación, Cultura y Deporte y de las universidades españolas determinará en buena medida el éxito de la estrategia UE-15. Mientras tanto, el sistema universitario haría bien en utilizar el activo más importante para el logro de ese objetivo de atracción del alumnado internacional, a fin de ser la cuna de una de las grandes culturas del mundo y el lugar de origen de la segunda lengua más internacional del mundo: la de habla española, con una comunidad de más de 450 millones de hablantes.

En España ya se viene haciendo un notable esfuerzo público y privado (Fundación Carolina y Universia, especialmente) para promover la movilidad de profesores y estudiantes, especialmente en el ámbito iberoamericano, pero ahí, sin duda, las universidades virtuales españolas tienen un gran papel que jugar y no solo en América, sino también en otras partes del orbe, como África o Asia.

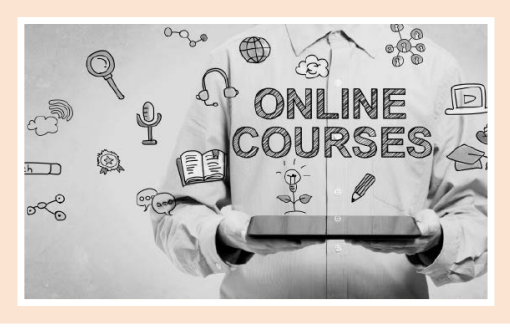

\section{Entre las razones por las que los estudiantes eligen estudiar en España está el idioma. Para atraer a esos alumnos internacionales las universidades españolas deben ofrecer cursos de español}

El ejemplo de la Open University británica es elocuente a este respecto, aunque también a ese propósito haya coadyuvado la implementación de otras estrategias, como la apertura de campus de universidades inglesas en las áreas geográficas de especial relevancia al respecto. En tal sentido, conviene no olvidar que el español es un activo muy importante para promocionar la internacionalización de la universidad española. El interés por aprender español crece en todo el mundo, especialmente en EE. UU. Entre las razones por las que los estudiantes eligen estudiar en España es el idioma. Para atraer a esos alumnos internacionales, las universidades españolas deben ofrecer cursos de español a lo largo de todo el año. De la misma manera, también deben ofertarse más cursos y programas de máster en inglés que estimulen a estudiantes que no saben el español a venir a España para después aprender nuestro idioma. Resulta paradójico que nuestro país, como potencia turística mundial, haya logrado ser el destino favorito de millones de personas, o que también por su calidad de vida sea el país preferido de los europeos para jubilarse, pero que, sin embargo, no lo sea para formarse y cursar estudios superiores.

Las universidades virtuales, con nuestros respectivos institutos de idiomas, estamos en disposición de coadyuvar decididamente en ese proceso en su doble vertiente, del alumnado y del profesorado, y además nos hallamos en estrecho contacto con la diplomacia cultural española y con el Instituto Cervantes, colaborando de esta forma a promover la imagen real de nuestro país por todo el mundo. Es, sin duda, solo un primer paso, pero muy importante, porque puede generar ese cambio necesario en la evolución del sistema, similar al del Instituto Británico con las universidades del Reino Unido. En esa apuesta más decidida por la internacionalización, el sistema universitario español no puede ignorar a las universidades virtuales y debe contar con ellas de una manera mucho más decidida. Estas pueden ofrecer a estudiantes de diferentes países programas formativos únicos con independencia de su lugar de residencia, multiplicándose además el número de proyectos de investigación internacionales, dado que la tecnología favorece la cooperación entre los miembros de distintos equipos. 
El potencial de profesores formados a través de una metodología on-line en distintos países y en contacto permanente a través de las mismas herramientas utilizadas para la formación virtual facilita la extensión de grupos y redes de investigación distribuidas por todo el mundo. Además, la experiencia de movilidad virtual puede servir extraordinariamente para preparar, dar seguimiento y complementar las experiencias de movilidad física de los estudiantes europeos más allá de las fronteras nacionales.

Las características de la formación on-line hacen posible que en una misma promoción coincidan alumnos de distintas nacionalidades que se encuentran residiendo en distintos países durante su periodo de formación. Ello le confiere a la formación on-line un carácter global del que deben ser conscientes estudiantes, profesores, instituciones y Administraciones. Sin duda, la posibilidad de compartir el proceso de estudio con personas de diferentes culturas permite conocer y comprender diferentes perspectivas y, por lo tanto, amplía y enriquece las posibilidades de aprendizaje del estudiante. En este sentido, cabe también destacar la mayor flexibilidad de la formación on-line en la configuración de nuevos itinerarios adaptados a las necesidades de un perfil específico de estudiantes. Por ejemplo, la colaboración interuniversitaria puede facilitar que sobre la base de una misma maestría virtual distintas universidades de diferentes países oferten una formación adaptada a las necesidades de su contexto social y cultural.

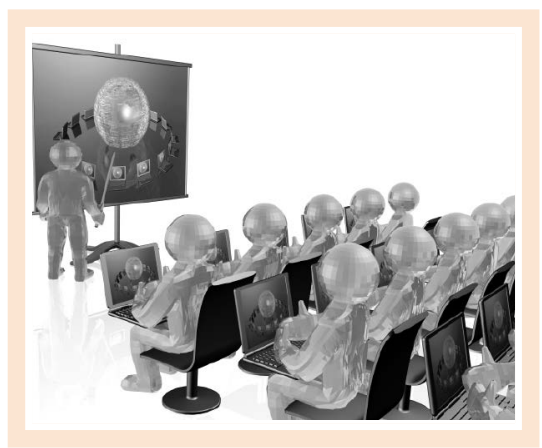

\author{
En esa apuesta más decidida por \\ la internacionalización, el sistema \\ universitario español no puede ignorar a \\ las universidades virtuales $y$ debe \\ contar con ellas de una manera mucho \\ más decidida. Estas pueden ofrecer \\ a estudiantes de diferentes países \\ programas formativos únicos con \\ independencia de su lugar de residencia, \\ multiplicándose además el número de \\ proyectos de investigación internacionales, \\ dado que la tecnología favorece la \\ cooperación entre los miembros de \\ distintos equipos
}

Pero también es necesario que esa aportación de las universidades virtuales al sistema universitario común se vea correspondida con la adecuada atención a sus especificidades 0 virtualidades más íntimas, lo que permitirá de paso atemperar algunos recelos en los procesos de acreditación de los títulos. En tal sentido, por ejemplo, la evaluación ha sido uno de los caballos de batalla en la defensa de la calidad de la formación on-line, razón por la cual las universidades virtuales son, si cabe, más escrupulosas que cualquier otra institución en el establecimiento de criterios, instrumentos y rúbricas de evaluación, así como en la identificación del estudiante y el control antiplagio.

Además, si consideramos la evaluación institucional, las características de la formación on-line permiten asimismo que se pueda realizar un seguimiento exhaustivo del desarrollo de un curso o una asignatura concreta, con una mucho mayor y mejor trazabilidad a la existente en las propias universidades presenciales. En este sentido, son claros los avances en la recogida y el análisis de datos dentro de lo que se viene a conocer como el learning analytics. También convendría exigir análogos estándares de calidad a aquellos grados o maestrías impartidos por universidades no virtuales en formato abierto con el fin de homogeneizar y amalgamar unos criterios comunes que debieran ser observados como garantía del buen funcionamiento del sistema en su conjunto. 